\title{
Combining clinical and MRI data to manage PI-RADS 3 lesions and reduce excessive biopsy
}

\author{
Shuo Yang, Wenlu Zhao, Shuangxiu Tan, Yueyue Zhang, Chaogang Wei, Tong Chen, Junkang Shen \\ Department of Radiology, The Second Affiliated Hospital of Soochow University, Suzhou, China \\ Contributions: (I) Conception and design: J Shen, S Yang; (II) Administrative support: J Shen, C Wei; (III) Provision of study materials or patients: \\ S Yang, S Tan, Y Zhang; (IV) Collection and assembly of data: S Yang, W Zhao, S Tan, Y Zhang; (V) Data analysis and interpretation: S Yang, W \\ Zhao, S Tan, T Chen; (VI) Manuscript writing: All authors; (VII) Final approval of manuscript: All authors. \\ Correspondence to: Junkang Shen. Department of Radiology, The Second Affiliated Hospital of Soochow University, No. 1055 Sanxiang Road, Gusu \\ District, Suzhou 215000, China. Email: 13962174116@163.com.
}

Background: Prostate Imaging Reporting and Data System version 2 (PI-RADS V2) 3 category lesions are of intermediate status with an equivocal risk of presenting clinically significant prostate cancer (csPCa). How to avoid excessive biopsies while improving the csPCa detection rate in these lesions has always been a clinical problem that needed to be solved. The purpose of this study is to explore the csPCa diagnostic value of clinical and magnetic resonance imaging (MRI) data for peripheral and transitional zone (PZ and TZ, respectively) PI-RADS 3 lesions to aid in clinical decision-making and reduce excessive biopsies.

Methods: From March 2016 to October 2018, a total of 629 men who underwent a prostate MRI and subsequently biopsy were enrolled. Two radiologists (with 3 and 7 years of experience, respectively) independently reviewed and scored all images using the PI-RADS V2 scoring criteria. Clinical and MRI data of men with PI-RADS 3 index lesions were collected by another radiologist. Univariate and multivariate analyses were performed to identify the risk factors of csPCa.

Results: In a subset of 121 men with 121 PI-RADS 3 index lesions, 25.6\% of the lesions (31/121) were PCa (Gleason score $\geq 6$ ), and $11.6 \%(14 / 121)$ were csPCa (Gleason score $\geq 7$ ). Further, $44.6 \%$ of lesions (54/121) were located in the PZ and 55.4\% (67/121) in the TZ. For PZ lesions, $18.5 \%$ of the lesions (10/54) were csPCa. Prostate-specific antigen density (PSAD) $(\mathrm{P}=0.024)$ and age $(\mathrm{P}=0.026)$ were independent risk factors for csPCa in the multivariate logistic analysis. The combination of PSAD and age yielded an area under the curve (AUC) value of 0.816 for predicting csPCa. If biopsy had been restricted to patients with a PSAD $\geq 0.15 \mathrm{ng} / \mathrm{mL}^{2}$ or an age $>68$ years, $24.1 \%$ (13/54) of patients would have avoided biopsy but only 1 (10\%) csPCa would have been missed, with a sensitivity of $80.0 \%$ and negative predictive value (NPV) of 92.3\%. For TZ lesions, only $6.0 \%$ of the lesions (4/67) were csPCa. The PSA and PSAD values in the PIRADS $3 \mathrm{TZ}$ lesions were higher in the csPCa group (45.07 and $0.47 \mathrm{ng} / \mathrm{mL}^{2}$, respectively) than in the noncsPCa group (10.03 and $0.17 \mathrm{ng} / \mathrm{mL}^{2}$, respectively).

Conclusions: CsPCa was detected at a relatively high rate in PI-RADS 3 PZ lesions. Combining PSAD and age could help to reduce excessive biopsies of such lesions. CsPCa is unlikely to be detected in PI-RADS 3 TZ lesions; thus, active surveillance may be an optimal choice for these lesions, especially among patients without high-risk factors.

Keywords: Prostate Imaging Reporting and Data System (PI-RADS); prostate cancer (PCa); multiparametric magnetic resonance imaging (mpMRI); risk stratification

Submitted Nov 17, 2019. Accepted for publication May 11, 2020.

doi: $10.21037 /$ tau-19-755

View this article at: http://dx.doi.org/10.21037/tau-19-755 


\section{Introduction}

Multiparametric magnetic resonance imaging (mpMRI) is currently accepted as the best imagological examination for prostate disease $(1,2)$. Prostate mpMRI used in suspected prostate cancer $(\mathrm{PCa})$ men can help improve the detection of clinically significant $\mathrm{PCa}$ (csPCa) and prevent unnecessary biopsies and treatments. To regulate and standardize MRI acquisition, image interpretation, and reporting of prostate MRI examinations, the European Society of Urogenital Radiology (ESUR) working group drafted the Prostate Imaging Reporting and Data System (PI-RADS) version 1 (V1) in 2012 (3) In 2015, a revised version (PI-RADS V2) based on this system was introduced (2). PI-RADS V2 use a 5 -point scoring system to evaluate lesions presenting csPCa. The likelihood of the presentation of csPCa increases as the score increases. Although PI-RADS V2 does not provide specified clinical management recommendations, a consensus has been reached that PI-RADS 1 or 2 lesions are csPCa-negative such that biopsy can be avoided, while PI-RADS 4 or 5 lesions are csPCa-positive and should be biopsied (4-6). For PI-RADS 3 lesions, however, it is still under debate whether a biopsy should be performed. PI-RADS V2 defines a category 3 lesion as a lesion with equivocal presence of csPCa. In previous studies, the proportions of PI-RADS index 3 lesions in all scored groups have ranged from $20 \%$ to $35 \%(7,8)$, and the overall csPCa detection rates in PI-RADS 3 lesions have ranged from $2 \%$ to $23 \%(6,9,10)$. In addition, almost three quarters of PCa lesions are located in the peripheral zone $(\mathrm{PZ})$ of the prostate, suggesting that the csPCa detection rate of PI-RADS 3 lesions in different prostatic zones may also be different $(9,11)$. The qualitative diagnosis of PI-RADS 3 lesions is difficult, and how to avoid excessive biopsies while improving the csPCa detection rate has always been a clinical problem that needed to be solved (12).

The purpose of this study was to explore the csPCa detection rates in PI-RADS 3 lesions in different prostatic zones, and to explore the clinical decision-making value of clinical and MRI data, helping to reduce excessive biopsies in patients with these lesions.

We present the following article in accordance with the STROBE reporting checklist (available at http://dx. doi. org/10. 21037/tau-19-755).

\section{Methods}

\section{Study population}

From March 2016 to October 2018, a total of 683 patients underwent a prostate MRI and subsequent biopsy at our center. The inclusion criteria for our study were as follows: (I) a prostate mpMRI examination and (II) transrectal ultrasound (TRUS)-guided " $10+X$ " systemic prostatic biopsy with or without a MR-TRUS targeted biopsy prior to a systemic biopsy. The exclusion criteria were as follows: (I) a PCa diagnosis and a history of therapy before MRI examination, (II) a 1.5-T MRI or other scannermediated prostate MRI examination and (III) incomplete mpMRI images or " $10+X$ " systemic biopsy. Ultimately, a total of 629 cases were retrospectively analyzed.

\section{MRI technique}

All scans were performed using a 3-T MRI scanner (Ingenia; Philips Healthcare, Best, The Netherlands) with a 32-channel body phased array coil as the receiving coil. All of the mpMRI sequences were set in accordance with the PI-RADS V2 recommendations. All scans included sagittal T2-weighted imaging (T2WI), axial T2WI, diffusionweighted imaging (DWI) (b values of $0,100,1,000$ and $2,000 \mathrm{sec} / \mathrm{mm}^{2}$ ) and dynamic contrast-enhanced MRI (DCE-MRI). The details of the imaging sequences, mainly including the sequence type, repetition time (TR), echo time (TE), field of view (FOV), matrix, slice thickness, slice gap, and number of signals averaged (NSA), are summarized in Table 1.

\section{Biopsy}

All patients underwent a TRUS " $10+X$ " systematic prostate biopsy. This biopsy approach divided the prostate into three sections (the base, the middle and the apex) from top to bottom, and each part was divided into the left and right regions. The right and left regions of the basal and middle parts were divided into inner and outer regions, and the whole prostate gland was divided into 10 areas. Each area was biopsied with 1 needle, and " $\mathrm{X}$ " more needles were then used to biopsy the suspicious area. 
Table 1 The details of the imaging sequence

\begin{tabular}{lrrrrrcc}
\hline Sequence & TR $(\mathrm{ms})$ & TE $(\mathrm{ms})$ & FOV $\left(\mathrm{mm}^{2}\right)$ & Matrix & Slice thickness $(\mathrm{mm})$ & Slice gap $(\mathrm{mm})$ & NSA \\
\hline Sagittal T2WI & $4,978.00$ & 100.00 & $240 \times 180$ & $240 \times 161$ & 1.50 & 0.15 & 2 \\
Axial T2WI & $3,000.00$ & 100.00 & $220 \times 220$ & $276 \times 238$ & 3.00 & 3 \\
Axial DWI & $6,000.00$ & 77.00 & $260 \times 260$ & $104 \times 125$ & 3.00 & 0.00 \\
DCE & 3.10 & 1.45 & $220 \times 220$ & $124 \times 121$ & 3.00 & 0.00 \\
\hline
\end{tabular}

TR, repetition time; TE, echo time; FOV, field of view; NSA, number of signals averaged; T2WI, T2-weighted imaging; DWI, diffusionweighted imaging; DCE, dynamic contrast-enhanced.

Some lesions underwent an MRI-targeted biopsy prior to a systematic biopsy. The histopathology of the prostate biopsies was reported by subspecialized uropathologists according to the 2014 International Society of Urologic Pathology guidelines (13). CsPCa was defined as a Gleason score $\geq 3+4$, and $\mathrm{PCa}$ was defined as a Gleason score $\leq 3+3$. We prioritized the pathology results of the target lesion if a lesion underwent an MRI-Targeted biopsy; if a lesion did not undergo an MRI-targeted biopsy, we used the pathology results of the above-mentioned subarea where the lesion was located. In our study, $24.8 \%$ of the lesions (30/121) underwent both targeted and systematic biopsies, while $75.2 \%$ (91/121) underwent only a systematic biopsy.

\section{Image analysis and data collection}

Two subprofessional genitourological radiologists (with 3 and 7 years of experience, respectively) performed blinded reviews of the 629 cases using the PI-RADS V2 criteria. For each case, the radiologists provided the PI-RADS V2 score of the index lesion until consensus was reached. Another radiologist (with 2 years of experience) collected the index lesion pathology according to the above-mentioned rules. For patients with PI-RADS 3 index lesions, the radiologist also collected the clinical characteristics, including the levels of serum prostate-specific antigen (PSA) and its derivatives [including free PSA (fPSA), free/total PSA (f/tPSA), and PSA density (PSAD)]; MRI characteristics, including the prostate volume (PV), lesion maximum diameter, and lesion location [the $\mathrm{PZ}$ or transitional zone (TZ) and the base, middle or apex]. The PV was calculated as the width $\times$ length $\times$ height $\times 0.52$ on T2-weighted images as recommended by the PI-RADS V2. The PSAD was equal to the PSA level divided by the PV.

\section{Statistical analysis}

First, descriptive statistics were used to analyze the clinical and MRI data. Then, a univariate logistic analysis and chisquare were performed to evaluate the difference between the csPCa group and the non-csPCa group. Multivariate logistic regression analysis was then performed to identify the independent risk factors of csPCa. Only those factors with $\mathrm{P}<0.15$ in the univariate logistic analysis were retained in the multivariate logistic analysis. A difference with $\mathrm{P}<0.05$ was considered to be significant in the multivariate logistic analysis. Finally, receiver operating characteristic (ROC) curve analysis was used to evaluate the csPCa predictive value of each risk factor and of combinations of risk factors. The above-mentioned statistical analyses were performed with IBM SPSS Statistics, version 20.0 (SPSS, Inc., Chicago, IL, USA).

\section{Results}

The detailed patient inclusion and exclusion follow charts are shown in Figure 1. A total of 629 patients met the inclusion and exclusion criteria. Of the 629 patients, $47.2 \%$ $(\mathrm{n}=297)$ were scored as PI-RADS 1 and 2, 20.0\% $(\mathrm{n}=126)$ were scored as PI-RADS 3, 16.9\% ( $\mathrm{n}=106)$ were scored as PI-RADS 4, and $15.9 \%(\mathrm{n}=100)$ were scored as PI-RADS 5; the csPCa detection rates were $1.7 \%, 11.1 \%, 44.3 \%$ and $82.0 \%$, respectively (Figure 2).

Of the 126 patients with PI-RADS 3 index lesions, 5 patients did not undergo a follow-up assessment due to incomplete clinical observation indexes. Ultimately, 121 patients with 121 PI-RADS 3 index lesions were enrolled for further statistical analyses. Of the lesions, $11.6 \%$ (14/121) were csPCa, $14.0 \%$ (17/121) were low-risk PCa 


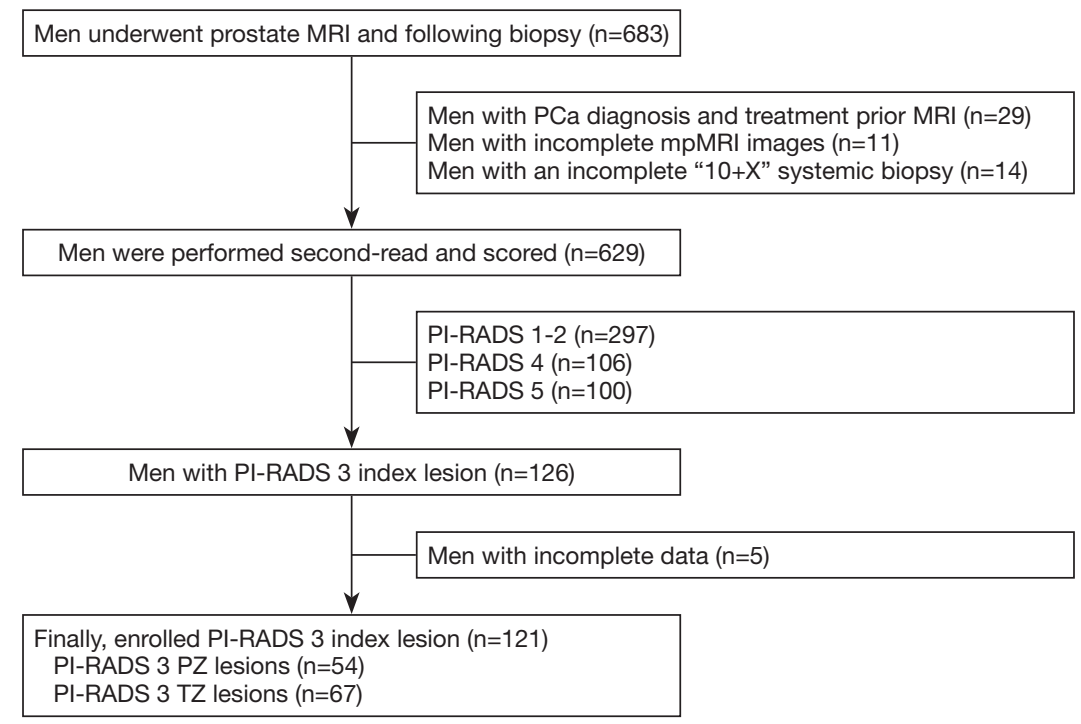

Figure 1 The detailed patient inclusion and exclusion follow charts. MRI, magnetic resonance imaging; PCa, prostate cancer; mpMRI, multiparametric MRI; PI-RADS, Prostate Imaging Reporting and Data System; PZ, peripheral zone; TZ, transitional zone.

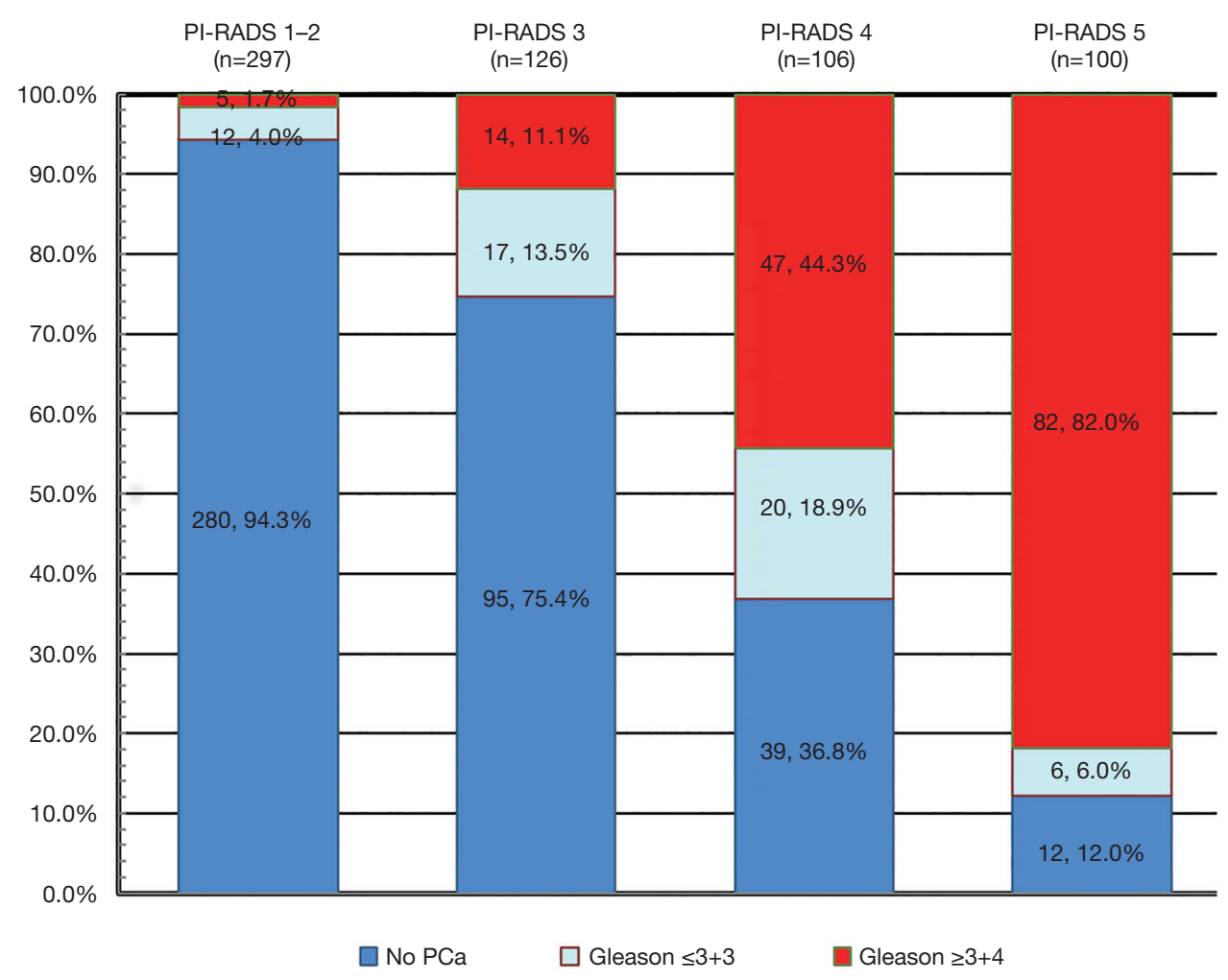

Figure 2 Pathology results of different PI-RADS V2 score group. PI-RADS V2, Prostate Imaging Reporting and Data System version 2; $\mathrm{PCa}$, prostate cancer. 
Table 2 Clinical and MRI data evaluated of peripheral lesions

\begin{tabular}{|c|c|c|c|}
\hline Clinical and MRI data & Non-clinically significant cancer $(n=44)$ & Clinically significant cancer $(n=10)$ & $P$ values \\
\hline Age (years), mean [SD] & $67[9]$ & $76[8]$ & $0.013^{\mathrm{a}}$ \\
\hline tPSA (ng/mL), median (IQR) & $8.39(5.52-13.89)$ & $14.28(7.89-20.27)$ & $0.111^{\mathrm{a}}$ \\
\hline fPSA (ng/mL), median (IQR) & $0.91(0.53-1.92)$ & $1.00(0.78-2.42)$ & $0.703^{\mathrm{a}}$ \\
\hline f/tPSA, median (IQR) & $0.12(0.93-1.88)$ & $0.09(0.05-0.14)$ & $0.182^{\mathrm{a}}$ \\
\hline PV (mL), median (IQR) & $39.01(29.78-69.45)$ & $36.66(24.94-46.58)$ & $0.122^{\mathrm{a}}$ \\
\hline Lesion diameter (mm), mean (SD) & $17.57(3.61)$ & $19.43(2.94)$ & $0.134^{\mathrm{a}}$ \\
\hline Lesion location, n (\%) & & & $0.817^{b}$ \\
\hline Base & $9(20.5)$ & $3(30.0)$ & \\
\hline
\end{tabular}

${ }^{\mathrm{a}}$, univariate cox regression analysis; ${ }^{\mathrm{b}}$, chi-square test. MRI, magnetic resonance imaging; $\mathrm{SD}$, standard deviation; IQR, interquartile range; tPSA, total prostate-specific antigen; fPSA, free PSA; f/tPSA, ratio of free-to-total PSA; PSAD, PSA density; PV, prostate volume.

and $74.4 \%(90 / 121)$ were benign. In addition, $44.6 \%$ of the lesions (54/121) were located in the PZ, while $55.4 \%$ (67/121) were located in the TZ.

\section{PZ}

Of the 54 PI-RADS 3 PZ lesions, 18.5\% (10/54) were lowrisk PCa, while $18.5 \%(10 / 54)$ were csPCa (including 5 with Gleason scores of 3+4, 2 with Gleason scores of $4+3$ and 3 with Gleason scores of $4+4)$. The univariate analysis results showed that PSAD $(\mathrm{P}=0.012)$, age $(\mathrm{P}=0.013)$, PSA $(\mathrm{P}=0.111), \mathrm{PV}(\mathrm{P}=0.122)$ and lesion diameter $(\mathrm{P}=0.134)$ were correlated with csPCa $(\mathrm{P}<0.15)$ (Table 2). Multivariate logistic regression analysis showed that a greater PSAD $(\mathrm{P}=0.024)$ and a greater age $(\mathrm{P}=0.026)$ were independent risk factors for predicting csPCa.

In the ROC curve analysis, a PSAD $\geq 0.15 \mathrm{ng} / \mathrm{mL}^{2}$ yielded a sensitivity of $80.0 \%$ and a specificity of $38.6 \%$ for predicting csPCa with an area under the curve (AUC) of 0.728 , and an age $>68$ years yielded a sensitivity of $80.0 \%$ and a specificity of $63.6 \%$ for predicting csPCa with an AUC of 0.766 . The combination of PSAD and age yielded an AUC of 0.816 , greater than the AUC of PSAD or age alone. If biopsy had been restricted to patients with a PSAD $\geq 0.15 \mathrm{ng} / \mathrm{mL}^{2}$ and age $>68$ years, the csPCa diagnostic sensitivity and specificity would have been $70.0 \%, 75.2 \%$, respectively, and biopsy would have been avoided in $66.7 \%$ of patients (36/54); however, $30 \%$ of csPCa cases $(3 / 10)$ would have been missed. If biopsy had been restricted to patients with a $P S A D \geq 0.15 \mathrm{ng} / \mathrm{mL}^{2}$ or an age $>68$ years, the csPCa diagnostic sensitivity and specificity, would have been $90.0 \%$ and $27.3 \%$, respectively, and biopsy would have been avoided in $24.1 \%$ of patients (13/54); in addition, only $10 \%$ of csPCa cases (1/10) would have been missed (Table 3$)$. Equivocal PZ lesions are shown in Figures 3,4.

\section{$T Z$}

Of the 67 PI-RADS 3 TZ lesions, 16.4\% (11/67) were PCa, while only $6.0 \%$ (4/67) were csPCa (including 3 with Gleason scores of $4+3$ and 1 with a Gleason score of $3+4)$, all of which were moderately differentiated adenocarcinomas. The csPCa group had a higher median PSA and PSAD and a lower f/tPSA than the non-csPCa group (45.07 vs. $10.03 \mathrm{ng} / \mathrm{mL}, 0.47$ vs. $0.17 \mathrm{ng} / \mathrm{mL}^{2}$, and 0.12 vs. 0.17 , respectively). However, the csPCa group had a higher mean $\mathrm{PV}$ than the non-csPCa group (81.71 vs. $50.00 \mathrm{~mL}$ ) (Table 4). Equivocal TZ lesions are shown in Figures 5,6.

\section{Discussion}

In this study, we found that PI-RADS 3 lesions were more likely to present in the $T Z(n=67)$ than in the PZ $(n=54)$. The csPCa detection date, however, was significantly lower in the $\mathrm{TZ}(6.0 \%, 4 / 67)$ than in the PZ $(18.5 \%, 10 / 54)$. For 
Table 3 Diagnostic value of PSAD, age and the combination for PI-RADS 3 index lesions of PZ

\begin{tabular}{|c|c|c|c|c|c|c|c|c|}
\hline Risk factors & AUC & $\begin{array}{l}\text { Decision } \\
\text { to biopsy }\end{array}$ & $\begin{array}{c}\text { Sensitivity } \\
(\%)\end{array}$ & $\begin{array}{c}\text { Specificity } \\
(\%)\end{array}$ & $\begin{array}{l}\text { PPV } \\
(\%)\end{array}$ & $\begin{array}{l}\text { NPV } \\
(\%)\end{array}$ & $\begin{array}{c}\text { Avoid biopsy, } \\
\%[n]\end{array}$ & $\begin{array}{c}\text { Missed csPCa, } \\
\%[n]\end{array}$ \\
\hline PSAD & 0.728 & $P S A D \geq 0.15$ & 80.0 & 38.64 & 22.9 & 89.5 & 35.2 [19] & 20.0 [2] \\
\hline \multirow[t]{2}{*}{ Combination } & 0.816 & $\begin{array}{c}P S A D \geq 0.15 \text { and } \\
\text { age }>68\end{array}$ & 70.0 & 75.2 & 38.9 & 83.3 & $66.7[36]$ & $30.0[3]$ \\
\hline & & $\begin{array}{c}P S A D \geq 0.15 \text { or } \\
\text { age }>68\end{array}$ & 90.0 & 27.3 & 22.0 & 92.3 & $24.1[13]$ & $10.0[1]$ \\
\hline
\end{tabular}

PSAD, prostate-specific antigen density; PI-RADS, Prostate Imaging Reporting and Data System; PZ, peripheral zone; AUC, area under the curve; PPV, positive predictive value; NPV, negative predictive value; csPCa, clinically significant prostate cancer.
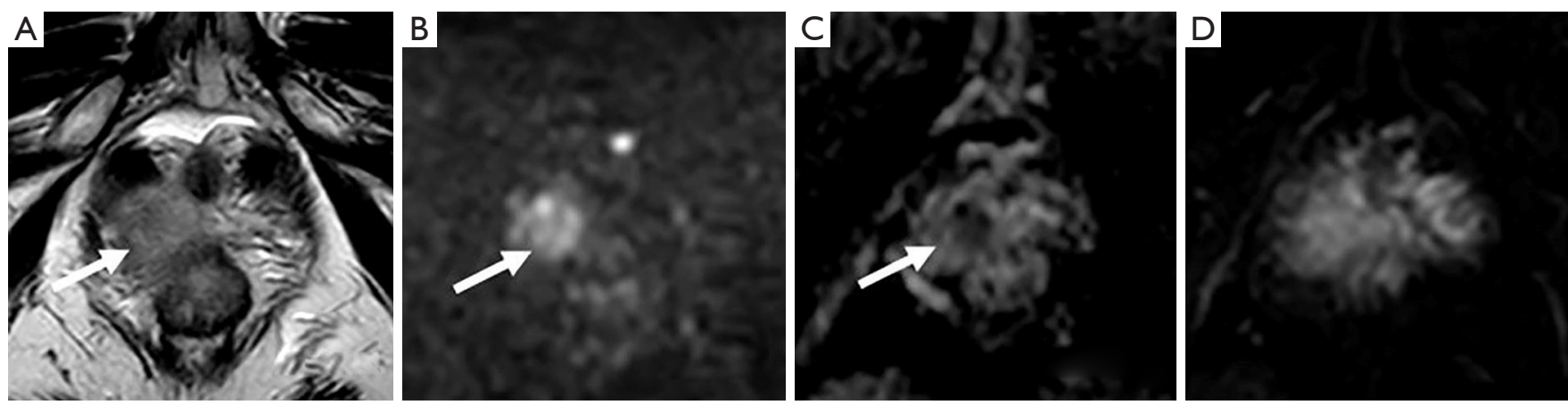

Figure 3 Patient with prostatitis after targeted biopsy, 76 years old, PSAD, $0.10 \mathrm{ng} / \mathrm{mL}^{2}$. The lesion is located in the right PZ of the apex part, and PI-RADS V2 score 3 was assigned (T2WI 3; DWI/ADC 3; DCE -). (A) Axial T2WI: rounded and moderate hypointensity, T2WI 3; (B) axial high-b-value DWI; (C) ADC map: focal moderately hyperintense on DWI and moderately hypointense on ADC, DWI/ADC 3; (D) axial early DCE-MRI: no focal early enhancement in corresponding parts, DCE -. Where the arrow points the lesion location. The white arrow indicates that there is no cancer in the lesion. PSAD, prostate-specific antigen density; PZ, peripheral zone; PI-RADS V2, Prostate Imaging Reporting and Data System version 2; T2WI, T2-weighted imaging; DWI, diffusion-weighted imaging; ADC, apparent diffusion coefficient; DCE, dynamic contrast-enhanced; MRI, magnetic resonance imaging.
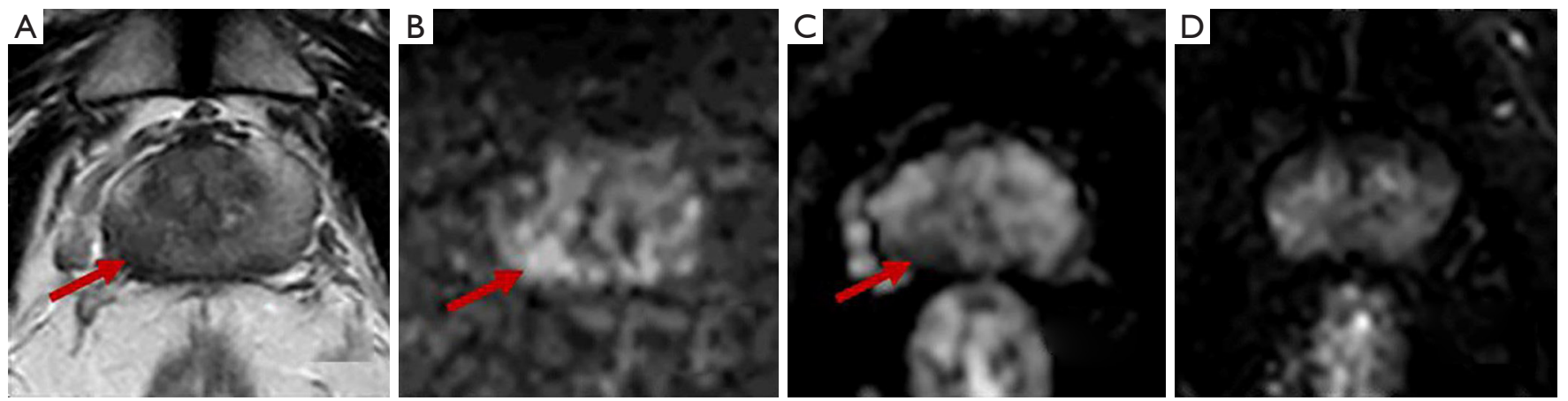

Figure 4 Patient with clinically significant PCa (Gleason score, 3+4), 80 years old, PSAD, $0.98 \mathrm{ng} / \mathrm{mL}^{2}$. The lesion is located in the right PZ of the middle part, and PI-RADS V2 score 3 was assigned (T2WI 3; DWI/ADC 3; DCE -). (A) Axial T2WI: homogeneous signal intensity with obscured margins, T2WI 3; (B) axial high-b-value DWI; (C) ADC map: focal moderately hyperintense on DWI and mildly to moderately hypointense on ADC, DWI/ADC 3; (D) axial early DCE-MRI: no focal early enhancement in corresponding parts, DCE -. Where the arrow points the lesion location. The red arrow indicates that there is cancer in the lesion. PCa, prostate cancer; PSAD, prostatespecific antigen density; PZ, peripheral zone; PI-RADS V2, Prostate Imaging Reporting and Data System version 2; T2WI, T2-weighted imaging; DWI, diffusion-weighted imaging; ADC, apparent diffusion coefficient; DCE, dynamic contrast-enhanced; MRI, magnetic resonance imaging. 
Table 4 Comparison of clinical and MRI data of transitional lesions

\begin{tabular}{|c|c|c|}
\hline Clinical and MRI data & Non-clinically significant cancer $(n=63)$ & Clinically significant cancer $(n=4)$ \\
\hline tPSA (ng/mL), median (IQR) & $10.03(5.20-17.28)$ & $45.07(17.19-60.85)$ \\
\hline fPSA (ng/mL), median (IQR) & $1.57(0.80-2.39)$ & $4.45(2.18-6.37)$ \\
\hline f/tPSA, median (IQR) & $0.17(0.11-0.22)$ & $0.12(0.08-0.21)$ \\
\hline PV (mL), median (IQR) & $50.00(35.00-69.90)$ & $81.71(53.88-126.75)$ \\
\hline Lesion diameter (mm), mean (SD) & $19.47(3.87)$ & $16.64(1.74)$ \\
\hline \multicolumn{3}{|l|}{ Lesion location, n (\%) } \\
\hline Base & $20(31.7)$ & 0 \\
\hline
\end{tabular}

MRI, magnetic resonance imaging; SD, standard deviation; IQR, interquartile range; tPSA, total prostate-specific antigen; fPSA, free PSA; f/tPSA, ratio of free-to-total PSA; PSAD, PSA density; PV, prostate volume.
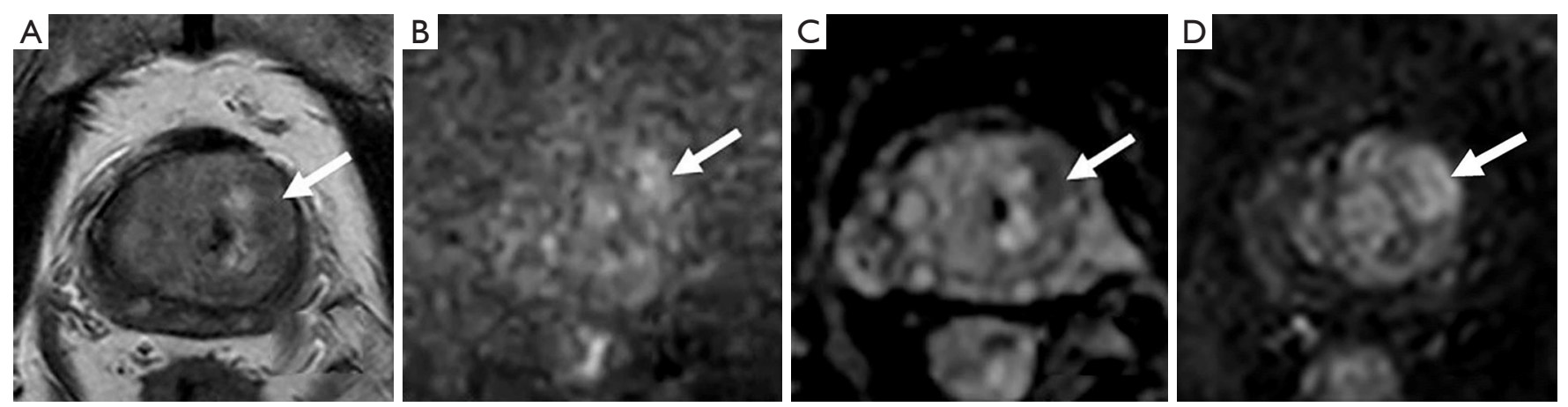

Figure 5 Patient with benign prostatic tissues, 81 years old, PSAD, $0.17 \mathrm{ng} / \mathrm{mL}^{2}$. The lesion is located in the left TZ of the middle part, and PI-RADS V2 score 3 was assigned (T2WI 3; DWI/ADC 3; DCE +). (A) Axial T2WI: heterogeneous signal intensity with obscured margins, T2WI 3; (B) axial high-b-value DWI; (C) ADC map: focal moderately hypointense on ADC and moderately hyperintense on DWI, DWI/ ADC 3; (D) axial early DCE-MRI: focal early enhancement in corresponding parts, DCE +. Where the arrow points the lesion location. The white arrow indicates that there is no cancer in the lesion. PSAD, prostate-specific antigen density; TZ, transitional zone; PI-RADS V2, Prostate Imaging Reporting and Data System version 2; T2WI, T2-weighted imaging; DWI, diffusion-weighted imaging; ADC, apparent diffusion coefficient; DCE, dynamic contrast-enhanced; MRI, magnetic resonance imaging.

PI-RADS 3 PZ lesions, older age and a higher PSAD were independent risk factors for csPCa. For PI-RADS $3 \mathrm{TZ}$ lesions, there was a low likelihood of csPCa, and high grade csPCa (Gleason $\geq 8$ ) was rarely detected.

The csPCa detection rate of equivocal lesions has significant variability in the reported studies. For example, Liddell et al. reported a generally lower detection rate of PCa in PI-RADS $3 \mathrm{PZ}$ and TZ lesions (3.9\% and $12.1 \%$, respectively) based on a modified PI-RADS scoring system (9). In contrast, Hansen et al. reviewed 143 PIRADS V2 3 category lesions with targeted and systemic biopsies, and found that the proportions of PI-RADS 3 lesions in the $\mathrm{PZ}$ and $\mathrm{TZ}$ were $41 \%$ and $59 \%$, respectively, both corresponding to csPCa detection rates of $21 \%$ (11). Recently, a meta-analysis by Schoots et al. reported an overall detection rate of csPCa of $17-20 \%$ based on 665 PI-RADS 3 lesions, which was higher than our results (6). Such huge variability may be related to the selection 

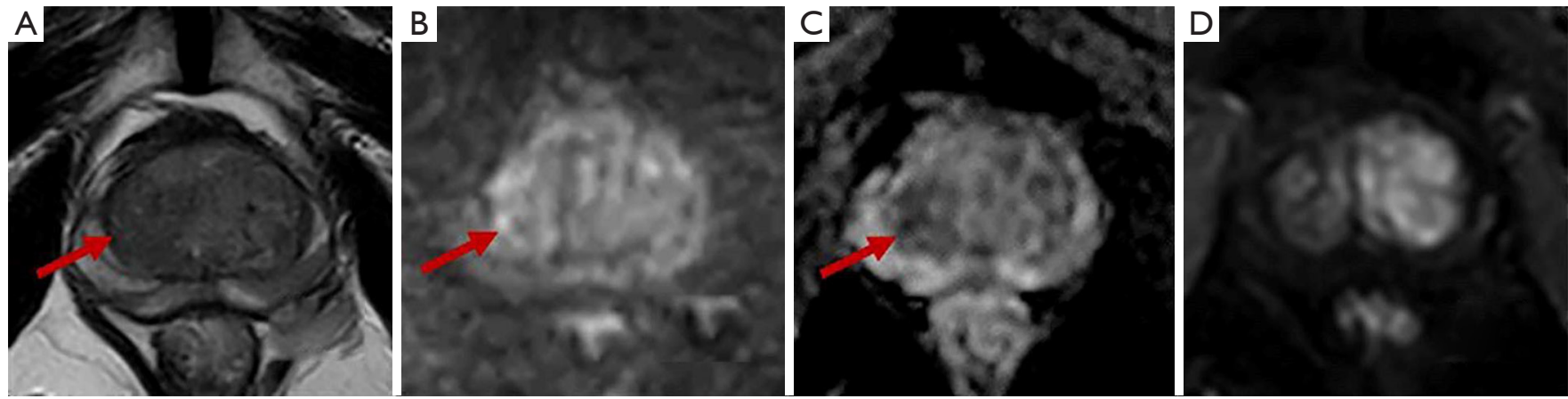

Figure 6 Patient with clinically significant PCa (Gleason score, 4+3), 64 years old, PSAD, $0.13 \mathrm{ng} / \mathrm{mL}^{2}$. The lesion is located in the right TZ of the middle part, and PI-RADS V2 score 3 was assigned (T2WI 3; DWI/ADC 3; DCE -). (A) Axial T2WI: heterogeneous signal intensity with obscured medial margins, T2WI 3; (B) axial high-b-value DWI; (C) ADC map: focal moderately hypointense on ADC and mildly hyperintense on DWI, DWI/ADC 3; (D) axial early DCE-MRI: no focal early enhancement in corresponding parts, DCE -. Where the arrow points the lesion location. The red arrow indicates that there is cancer in the lesion. PCa, prostate cancer; PSAD, prostate-specific antigen density; TZ, transitional zone; PI-RADS V2, Prostate Imaging Reporting and Data System version 2; T2WI, T2-weighted imaging; DWI, diffusion-weighted imaging; ADC, apparent diffusion coefficient; DCE, dynamic contrast-enhanced; MRI, magnetic resonance imaging.

criteria of the study populations. In our study, only 30 lesions underwent both targeted and systemic biopsies, and the overall csPCa detection rate was $16.7 \%(5 / 30)$ in this group, which was approximate to the averages. Besides, our results showed that there was a higher detection rate of csPCa in the PI-RADS $3 \mathrm{PZ}$ lesions (18.5\%) than the TZ lesions $(6.0 \%)$, which may again reflect the fact that $\mathrm{PCa}$ is common in the $\mathrm{PZ}$.

Given that the lesions in different zones had significantly different csPCa detection rates, we performed analyses according to the lesion zone. For PI-RADS 3 PZ lesions, PSAD and age were independent risk factors for predicting csPCa, with AUC values of 0.728 and 0.766 , respectively. The combination of PSAD and age yielded an AUC of 0.816 for predicting csPCa. If biopsy had been restricted to those with a PSAD $\geq 0.15 \mathrm{ng} / \mathrm{mL}^{2}$ and an age $>68$ years, the csPCa detection rate would have improved to $38.9 \%$ with a sensitivity of $70.0 \%$. If biopsy had been restricted to the patients with a PSAD $\geq 0.15 \mathrm{ng} / \mathrm{mL}^{2}$ or an age $>68$ years, it could have yielded a sensitivity of $90.0 \%$, a negative predictive value (NPV) of $92.3 \%$ and a csPCa detection rate of $22.0 \%$, while allowing biopsies to be avoided in $24 \%$ of patients (13/54); only $10 \%$ of csPCa cases $(1 / 10)$ would have been missed. Although the csPCa detection rate was not ideal, the latter biopsy criteria had sufficient sensitivity and maximally avoided csPCa missed.

Nowadays, PSAD and age have been widely validated as independent predictors of csPCa; however, data in men with PI-RADS 3 lesions are limited and controversial. For PSAD, Tan et al. in their enrolled group of 70 Southeast Asian men found that PSAD and prostate health index (PHI) were the best predictors of csPCa, with AUC values of 0.61 and 0.77 , respectively, and a PSAD $\geq 0.10 \mathrm{ng} / \mathrm{mL}^{2}$ would have detected all but one tumor, with a NPV of $93 \%$ and a sensitivity of $92 \%$ (14). Ullrich et al. found that only $\mathrm{PV}$ was a significantly different predictor $(\mathrm{P}=0.015)$, but PSAD had no statistical significance $(\mathrm{P}=0.31)$, although it was higher in patients with $\mathrm{PCa}$ than in those without. The author concluded that clinical risk factors such as PSA and PSAD must be considered in decision-making for PI-RADS 3 lesions (15). For age, Sheridan et al. calculated three risk factors of csPCa: age $>70$ years, $\mathrm{PV}<36 \mathrm{~mL}$ and positive DRE in their multivariate analysis, and PI-RADS 3 lesions associated with two or more risk factors were accurately predicted, with a sensitivity of $53 \%$ and a specificity $95 \%$ (16). However, those above-mentioned studies on PIRADS 3 lesions did not analyze the lesions by zone. In our study, based on PI-RADS 3 PZ lesions, we observed that a clinical threshold of PSAD $\geq 0.15 \mathrm{ng} / \mathrm{mL}^{2}$ and a calculated threshold of age $>68$ years were the best predictors of csPCa.

For PI-RADS $3 \mathrm{TZ}$ lesions, only $4 \mathrm{csPCa}$ cases were detected with a detection rate of $6.0 \%$, including 3 cases with a Gleason score of $4+3$ and 1 case with a Gleason of $3+4$. Therefore, univariate and multivariate analyses were not performed for PI-RADS $3 \mathrm{TZ}$ lesions because of the small sample of csPCa cases. However, the csPCa cases had 
a higher median PSA and PSAD and a lower median f/tPSA than the non-csPCa cases. Felker et al. analyzed 90 cases with 96 PI-RADS 3 lesions in the TZ, and observed that the combination of a PSAD of $0.15 \mathrm{ng} / \mathrm{mL}^{2}$ or greater and an apparent diffusion coefficient (ADC) value of less than $1,000 \mathrm{~mm}^{2} / \mathrm{s}$ could yield an AUC of 0.91 for $\mathrm{csPCa}$ (17). In our study, if biopsy had been restricted to patients with a PSAD $\geq 0.15 \mathrm{ng} / \mathrm{mL}^{2}, 44.8 \%$ (30/67) of patients would have avoided a biopsy but only 1 case of csPCa would have been missed. If biopsy had been restricted to patients with a PSA $\geq 10 \mathrm{ng} / \mathrm{mL}^{2}, 47.8 \%$ (32/67) of patients would have avoided a biopsy but only 1 case of csPCa would have been missed. Unlike other studies, the csPCa group in this study had a higher mean PV than the non-csPCa group $(15,18)$, which may have been due to the low number of csPCa cases. We found that one of the csPCa cases had a PV of $135 \mathrm{~mL}$ because of obvious benign prostatic hyperplasia $(\mathrm{BPH})$ and pathologically confirmed acute inflammatory edema.

Our study has several limitations. First, this was a retrospective, single-center study, that had relatively few PI-RADS 3 lesions enrolled, especially csPCa lesions of the TZ, which may have resulted in sampling bias. Second, compared with other studies, our study included only a few lesions $(24.8 \%, 30 / 121)$ that underwent both targeted and system biopsies, which may have increased the risk of an incorrect biopsy. Previous studies have concluded that targeted biopsies have a higher csPCa detection rate than standard biopsies $(19,20)$, which also explains why our study had a slightly lower csPCa detection rate than that observed in meta-analyses (6). Third, our study included a small amount of clinical and MRI data, and we failed to find MRI data that could predict csPCa. However, these data are common and easily accessible, and our primary aim was to provide a simple and practical predictive model for clinical reference.

\section{Conclusions}

Our results have shown that the detection rate of csPCa for PI-RADS $3 \mathrm{PZ}$ lesions (18.5\%) is significantly higher than that for TZ lesions (6.0\%). For PI-RADS $3 \mathrm{PZ}$ lesions, risk stratification with the combination of PSAD and age may be useful for selecting whether or not to perform a biopsy. For PI-RADS 3 TZ lesions, given the low csPCa detection rate and the relatively minimal histological differences, we suggest that active surveillance may be the optimal choice for patients, especially patients without high risk factors, such as a PSA $<10 \mathrm{ng} / \mathrm{mL}$ or
PSAD $<0.15 \mathrm{ng} / \mathrm{mL}^{2}$. Larger-scale and multicenter trials are necessary to check the accuracy and replicability of our results. Further, incorporating these risk factors into future PI-RADS 3 scoring criteria may be useful for increasing the diagnostic confidence of equivocal lesions.

\section{Acknowledgments}

We thank AME Editing Service for editing this manuscript. Funding: This study was supported by financial grants from the National Natural Science Foundation of China (grant no. 81801754).

\section{Footnote}

Reporting Checklist: The authors have completed the STROBE reporting checklist. Available at http://dx. doi. org/10. 21037/tau-19-755

Data Sharing Statement: Available at http://dx. doi. org/10. 21037/tau-19-755

Conflicts of Interest: All authors have completed the ICMJE uniform disclosure form (available at http://dx. doi. org/10. 21037/tau-19-755). The authors have no conflicts of interest to declare.

Ethical Statement: The authors are accountable for all aspects of the work in ensuring that questions related to the accuracy or integrity of any part of the work are appropriately investigated and resolved. This retrospective study was approved by the Institutional Ethical Committee of our hospital, which waived the requirement for written informed consent.

Open Access Statement: This is an Open Access article distributed in accordance with the Creative Commons Attribution-NonCommercial-NoDerivs 4.0 International License (CC BY-NC-ND 4.0), which permits the noncommercial replication and distribution of the article with the strict proviso that no changes or edits are made and the original work is properly cited (including links to both the formal publication through the relevant DOI and the license). See: https://creativecommons.org/licenses/by-nc-nd/4.0/.

\section{References}

1. Kasel-Seibert M, Lehmann T, Aschenbach R, et al. 
Assessment of PI-RADS v2 for the Detection of Prostate

Cancer. Eur J Radiol 2016;85:726-31.

2. Weinreb JC, Barentsz JO, Choyke PL, et al. PI-RADS Prostate Imaging-Reporting and Data System: 2015, Version 2. Eur Urol 2016;69:16-40.

3. Barentsz JO, Richenberg J, Clements R, et al. ESUR prostate MR guidelines 2012. Eur Radiol 2012;22:746-57.

4. Grey AD, Chana MS, Popert R, et al. Diagnostic accuracy of magnetic resonance imaging (MRI) prostate imaging reporting and data system (PI-RADS) scoring in a transperineal prostate biopsy setting. BJU Int 2015;115:728-35.

5. Wysock JS, Mendhiratta N, Zattoni F, et al. Predictive value of negative $3 \mathrm{~T}$ multiparametric magnetic resonance imaging of the prostate on 12-core biopsy results. BJU Int 2016;118:515-20.

6. Schoots IG. MRI in early prostate cancer detection: how to manage indeterminate or equivocal PI-RADS 3 lesions? Transl Androl Urol 2018;7:70-82.

7. Hansen NL, Barrett T, Kesch C, et al. Multicentre evaluation of magnetic resonance imaging supported transperineal prostate biopsy in biopsy-naive men with suspicion of prostate cancer. BJU Int 2018;122:40-9.

8. Shin T, Smyth TB, Ukimura O, et al. Diagnostic accuracy of a five-point Likert scoring system for magnetic resonance imaging (MRI) evaluated according to results of MRI/ultrasonography image-fusion targeted biopsy of the prostate. BJU Int 2018;121:77-83.

9. Liddell H, Jyoti R, Haxhimolla HZ. mp-MRI Prostate Characterised PIRADS 3 Lesions are Associated with a Low Risk of Clinically Significant Prostate Cancer-A Retrospective Review of 92 Biopsied PIRADS 3 Lesions. Curr Urol 2015;8:96-100.

10. Hermie I, Van Besien J, De Visschere P, et al. Which clinical and radiological characteristics can predict clinically significant prostate cancer in PI-RADS 3 lesions? A retrospective study in a high-volume academic center. Eur J Radiol 2019;114:92-8.

11. Hansen NL, Koo BC, Warren AY, et al. Sub-differentiating equivocal PI-RADS-3 lesions in multiparametric magnetic resonance imaging of the prostate to improve cancer

Cite this article as: Yang S, Zhao W, Tan S, Zhang Y, Wei C, Chen T, Shen J. Combining clinical and MRI data to manage PI-RADS 3 lesions and reduce excessive biopsy. Transl Androl Urol 2020;9(3):1252-1261. doi:10.21037/tau-19-755 detection. Eur J Radiol 2017;95:307-13.

12. Gupta RT, Mehta KA, Turkbey B, et al. PI-RADS: past, present, and future. J Magn Reson Imaging 2019. doi: 10.1002/jmri.26896.

13. Egevad L, Delahunt B, Evans AJ, et al. International Society of Urological Pathology (ISUP) Grading of Prostate Cancer. Am J Surg Pathol 2016;40:858-61.

14. Tan TW, Png KS, Lee CH, et al. MRI fusion-targeted transrectal prostate biopsy and the role of prostate-specific antigen density and prostate health index for the detection of clinically significant prostate cancer in Southeast Asian men. J Endourol 2017;31:1111-6.

15. Ullrich T, Quentin M, Arsov C, et al. Risk stratification of equivocal lesions on multiparametric magnetic resonance imaging of the prostate. J Urol 2018;199:691-8.

16. Sheridan AD, Nath SK, Syed JS, et al. Risk of clinically significant prostate cancer associated with prostate imaging reporting and data system category 3 (equivocal) lesions identified on multiparametric prostate MRI. AJR Am J Roentgenol 2018;210:347-57.

17. Felker ER, Raman SS, Margolis DJ, et al. Risk stratification among men with prostate imaging reporting and data system version 2 category 3 transition zone lesions: is biopsy always necessary? AJR Am J Roentgenol 2017;209:1272-7.

18. Radtke JP, Wiesenfarth M, Kesch C, et al. Combined clinical parameters and multiparametric magnetic resonance imaging for advanced risk modeling of prostate cancer-patient-tailored risk stratification can reduce unnecessary biopsies. Eur Urol 2017;72:888-96.

19. Ahmed HU, El-Shater Bosaily A, Brown LC, et al. Diagnostic accuracy of multi-parametric MRI and TRUS biopsy in prostate cancer (PROMIS): a paired validating confirmatory study. Lancet 2017;389:815-22.

20. Radtke JP, Kuru TH, Bonekamp D, et al. Further reduction of disqualification rates by additional MRItargeted biopsy with transperineal saturation biopsy compared with standard 12-core systematic biopsies for the selection of prostate cancer patients for active surveillance. Prostate Cancer Prostatic Dis 2016;19:283-91. 